\title{
RESEARCH CONCERNING THE DISTRIBUTION OF TEMPERATURE IN ASPHALT MIXTURES WITH THE HELP OF THERMO-PHYSICAL CHARACTERISTICS FOR LABORATORY TESTS
}

Marc Paul- Assistant Professor, Ph.D., University "Politehnica" of Timişoara, Civil Engineering Faculty, CCTFC Department, paul_marc22@yahoo.com

Costescu Ciprian- Senior Lecturer, Ph.D., University "Politehnica" of Timişoara, Civil Engineering Faculty, CCTFC Department, ciprian.costescu@ct.upt.ro

\section{Abstract:}

The characterization of the material through laboratory tests performed on asphalt mixtures offers significant performance predictions for asphalt pavements only when the material temperature is correctly taken into consideration. This paper presents an analytical pattern which, based on the thermo-physical characteristics, can predict correctly the distribution of temperatures in the mass of asphalt mixtures.

The paper also presents the organizational structure of laboratory testing, designed to validate the model for temperature determination. The temperature measurements are realized on a range of cylindrical samples at different moments in time, after submitting the sample from a low balance condition to a high balance condition by placing it in the environment precinct, according to the future test.

In conclusion, the paper aims at realizing and developing a model as a practical instrument able to offer a reliable assessment concerning the time required in order to reach the testing temperature specified for the samples of asphalt mixtures for different laboratory trials.

Key words: thermo-physical characteristics/testing temperature/final time

\section{INTRODUCTION}

The paper presents a laboratory research on the temperature distribution in asphalt mixtures. It shows the time required by asphalt mixture samples to reach the correct temperature for the tests they are subjected to, so that there is no great difference in value between the superior and the inferior limit stipulated by the standard for the same asphalt mixture dosage. Thus, with the help of thermophysical characteristics, the complex development processes can be followed 
during subjecting the asphalt mixture samples to high temperatures and to low temperatures over the entire life span and the laboratory analysis time can be reduced.

This identification of the thermo-physical characteristics is made possible by the development of technical applications of fluxmetric measurement, which allow the definition of simple modalities of plan thermal status.

The behavior and characterization of asphalt mixtures is one of the present issues emerging from the high degree of variability in the composition of the mixture, from the weather, hydrological and traffic conditions. In order to research the experiment and measurement of the characteristic features of asphalt mixtures, some non-destructive, reliable and precise methods are needed, since these characteristics vary in time and cannot always be described rigorously by simple relations.

The mechanic behavior of asphalt mixtures depends largely on the temperature. This is why their behavior extends with the increase of the temperature and contracts with the decrease of the temperature, the results being interpreted as thermal cracking. At high temperatures, the asphalt mixture is relatively soft and sensitive to rutting (permanent strain), in accordance to repeated traffic loadings, while at low temperatures it is relatively fragile and sensitive to thermal cracking. These ample research reasons determined the realization of measurements to predict the temperature of the pavement in situ, this being the basis of different models and applications concerning the anticipation of the variation in time of the temperature in the asphalt mixture.

The thermal characteristics of materials have a significant impact on the distribution of temperature and its variation in a body. These characteristics are required as input for the modeling of the relation time - temperature in any solid body, such as the asphalt mixture.

The thermo-physical characteristics of asphalt mixtures, such as the thermal conductivity, the specific heat, the diffusibility and the heat capacity, have been researched during a training period I had in France, with the help of fluxmetric measurements.

\section{EXPERIMENTAL PROGRAM}

In the laboratory, the cylindrical samples are usually the most used for different tests to determine the physic-mechanical characteristics. This paper presents the 
study concerning the distribution and the validation of the model temperature on a standard cylindrical sample. After compaction with the gyratory compactor, the sample was prepared for placing the temperature thermocouples. They were placed on the upper side of the cylinder surface, on the side surface and on the inside in $10 \mathrm{~mm}, 25 \mathrm{~mm}, 40 \mathrm{~mm}$ and $50 \mathrm{~mm}$ deep slots on the side faces. An important characteristic of this type of asphalt concrete is that the bitumen content is $4,9 \%$, the voids are $5,8 \%$ and the density is $2440 \mathrm{~kg} / \mathrm{m}^{3}$.

In order to have the testing temperature under control, the sample was introduced in a environmental measuring chamber. The temperature in the inside of the testing chamber was controlled by connecting it to a thermostat bath of the heat exchanger placed in an adjacent plan to the testing chamber. The difference of the air temperature between the testing chamber and the plan resulted with variations of $\pm 0,001{ }^{\circ} \mathrm{C}$.

The main goal of the experiment is to monitor the temperature changes in time in different points of the asphalt concrete sample, before being subjected to physical-mechanical tests. In order to reach this goal, the cylindrical sample was conditioned to a specific temperature of $3.5^{\circ} \mathrm{C}$ for 10 hours, in order to ensure the uniform distribution of temperature in a balance condition of $36^{\circ} \mathrm{C}$. In order to minimize the loss of temperature during displacement, the sample was wrapped in a cloth which had been kept at the same temperature as the sample. Once the sample introduced in the testing chamber and the thermocouples connected, the gathering of data began.

\section{GENERATION EQUATIONS}

The transfer process in a solid is described by the energy transitory equation [1]. In the Cartesian tridimensional system the equation is written as follows:

$$
k_{x} \frac{\partial^{2} T}{\partial x^{2}}+k_{y} \frac{\partial^{2} T}{\partial y^{2}}+k_{z} \frac{\partial^{2} T}{\partial z^{2}}+\frac{d^{3} u}{d t^{3}}=\rho c \frac{\partial T}{\partial t}
$$

where: $\quad T$ - temperature;

$k_{x}, k_{y}, k_{z}$ - thermal conductivity;

$x, y, z$ - directions of the respective coordinates;

$\rho$ - density of the sample;

$c$ - specific heat;

$u$ - internal energy generated on the volume unit;

$t$ - time.

For the tridimensional cylindrical coordinate system, the equation results as follows: 


$$
k_{r} \frac{\partial}{\partial_{r}}\left(r \frac{\partial T}{\partial r}\right)+k_{\varphi} \frac{1}{r^{2}} \frac{\partial^{2} T}{\partial \varphi^{2}}+k_{z} r \frac{\partial^{2} T}{\partial z^{2}}+\frac{d^{3} u}{d t^{3}}=\rho c \frac{\partial T}{\partial t}
$$

where: $\quad k_{r}, k_{\varphi}, k_{z}$ - thermal conductivity, $r, \varphi, z$ - spatial coordinates on respective directions.

In order to determine as correctly as possible the temperature variations on the cylindrical sample, its position is considered in the testing chamber at the intersection between an infinite plan with known widths and an infinite cylinder with known radius, according to figure 1 . Sometimes, to reduce friction on the contact surface between the plan wall and the sample, a Teflon disk can be used at the bottom during the measurement. Depending on the thickness and the physical-thermal characteristics, the sample could become insulating at the bottom and in this case it could be considered as the intersection of half plan wall with an infinite cylinder.

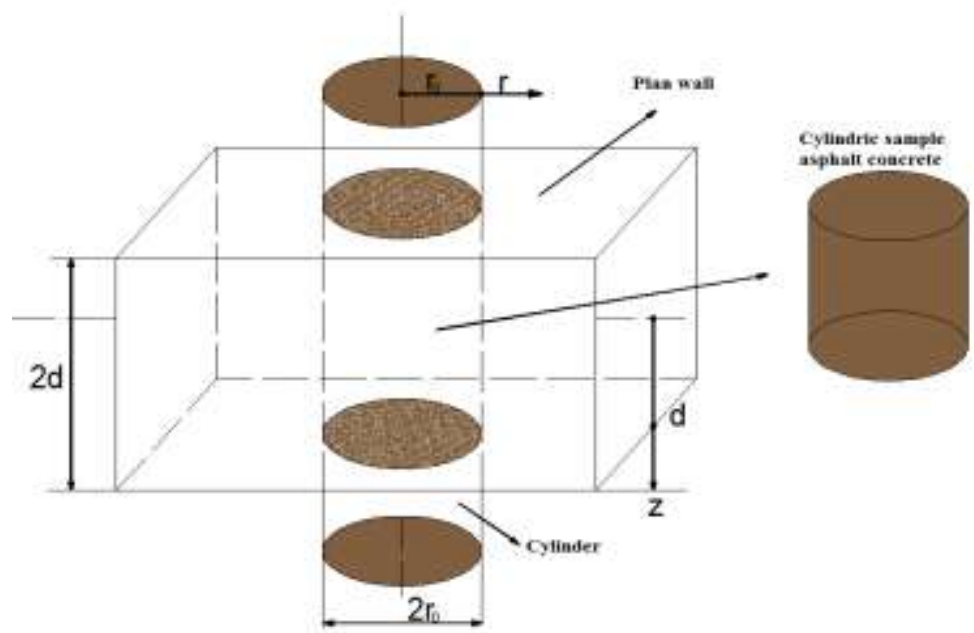

Figure 1. Intersection between the cylindrical sample and the infinitely plan wall

In the case when the thermal conductivity is supposed to be uniform and since there is no heat generation inside the asphalt concrete sample, the differential equation (1) for an infinitely plan wall can be simplified as follows:

$\frac{\partial^{2} T}{\partial z^{2}}=\frac{\rho c}{k} * \frac{\partial T}{\partial t}=\frac{1}{\alpha} * \frac{\partial T}{\partial t}$

$\alpha=\frac{k}{\rho c} \rho c$ - thermal diffusivity of the asphalt concrete;

- for the cylinder the equation (2) can be simplified as follows:

$\frac{1}{r} \frac{\partial}{\partial r}\left(r \frac{\partial T}{\partial r}\right)=\frac{1}{\alpha} \frac{\partial T}{\partial t}$ 
Considering Fourier's law, the adimensional size of the heat conduction is expressed as follows: $F_{0}=\frac{\alpha t}{d_{0}^{2}}$, and the Biot number is expressed $B i=\frac{h d_{0}}{k},\left(d_{0}\right.$ is half of the thickness of the plan wall, or the cylinder radius).

The temperature is always normalized for the proposed solutions:

- for the plan wall, $\theta\left(z, F_{0}\right)=\frac{T(z, t)-T_{\infty}}{T_{0}-T_{\infty}}$;

- for the cylinder, $\theta\left(R, F_{0}\right)=\frac{T(r, t)-T_{\infty}}{T_{0}-T_{\infty}}$.

The depth is normalized as $R=\frac{r}{r_{0}}$, where $r_{0}$ the radius of the cylinder, therefore the equations ( 3 ) and (4) can be written as follows:

$$
\begin{aligned}
& \frac{\partial^{2} \theta}{\partial Z^{2}}=\frac{\partial \theta}{\partial F_{0}} \\
& \frac{1}{R} \frac{\partial}{\partial R}\left(R \frac{\partial \theta}{\partial R}\right)=\frac{\partial \theta}{\partial F_{0}}
\end{aligned}
$$

In the case of this experiment, the conduction heat $h$ was considered as invariable due to the invariable temperature in the testing chamber, therefore the solutions to the equations (5) and (6) can be approximated by using the method of variable separation, the following relations result for the cylinder [5], [6]:

$$
\begin{aligned}
& \theta\left(R, F_{0}\right)=\sum_{n=1}^{\infty} A_{n} e^{-\lambda_{n}^{2} F_{0}} J_{0}\left(\lambda_{n} \frac{r}{r_{0}}\right) \\
& \lambda_{n} \frac{J_{1}\left(\lambda_{n}\right)}{J_{0}\left(\lambda_{n}\right)}=B_{i} \text { ssi } A_{n}=\frac{2 J_{1}\left(\lambda_{n}\right)}{\lambda_{n}\left[J_{0}^{2}\left(\lambda_{n}\right)+J_{1}^{2}\left(\lambda_{n}\right)\right]}
\end{aligned}
$$

where: $J_{0}\left(\lambda_{n}\right) s i J_{1}\left(\lambda_{n}\right)$ are the first two terms of the Bessel function.

The normalized temperature $\theta(z, r, t)$ for the cylinder of $(z, r)$ coordinates and the time $t$, can be expressed in this experiment as a multiplication of results for an infinitely plan wall and cylinder, as follows:

$$
\theta(z, r, t)=\theta(z, t)_{\text {plan }} \times \theta(r, t)_{c i l i n d r u}
$$

If $F_{0}>0,2$, the equation (7) can be expressed in a single term $(n=1)$, which results from precision in $2 \%$ proportion [2].

Depending on the values taken by the parameters $\lambda$ and $A$ [7], the equation (8) can be solved by using the Newton-Raphson method.

The thermo-physical characteristics for the asphalt concrete were directly measured during another experiment realized in France.

\section{RESULTS AND DISCUSSION}

The measured values presented in figure 2 show that in the asphalt concrete sample the temperature increases suddenly in different points, even from the beginning of the measurement process, then it continues with a slow 
increase; it results in an extended period of time in reaching a condition of temperature balance. The temperature at the upper surface increases the most rapidly; as a point of interest it is worth mentioning that the temperature gradient stays constant as it gets closer to the center of the sample.

In the end, in order to have an adequate time, all the points in the model need to show an increase to the balance temperature, being at the same level with the environmental temperature. The anticipations presented in figure 3 are based on the thermal characteristics determined for the respective dosage of asphalt concrete. Once the initial and final temperatures have been settled, the temperature in any moment depends on three parameters: thermal conductivity $k$, specific heat $c$ and convection heat $h$.

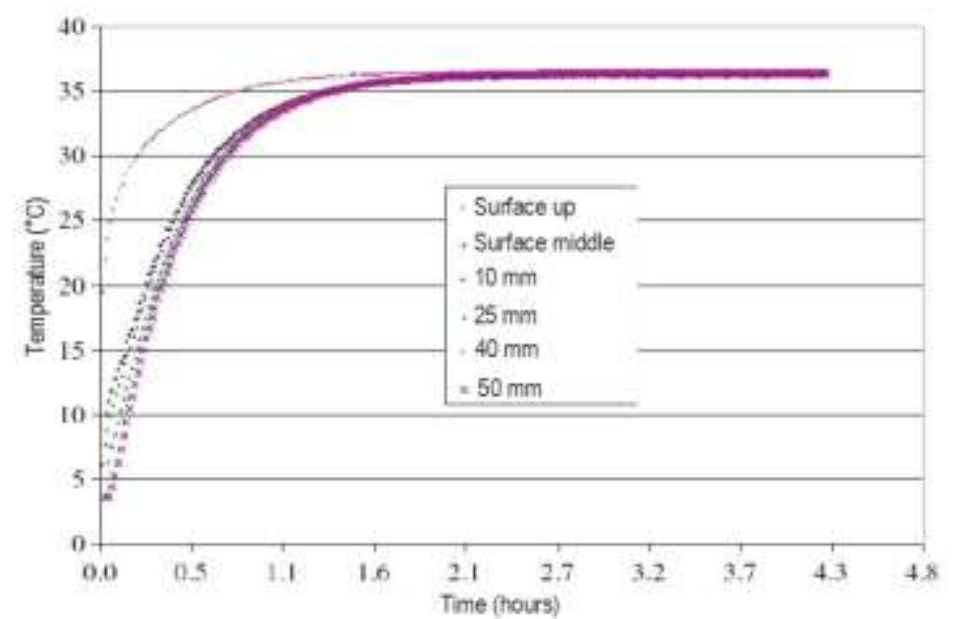

Figure 2. Measured temperature

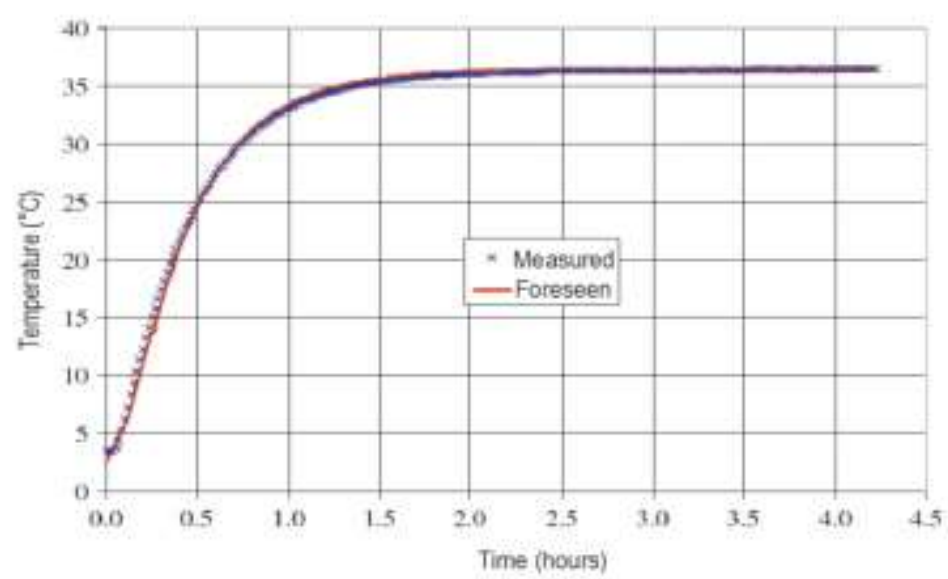

Figure 3. Measured temperature compared to its prediction in the center of the sample using thermo-physical characteristics 
In order to emphasize the impact of the thermo-physical characteristics on the distribution of temperature in the mass of asphalt concrete, the thermal conductivity was considered variable from $1.0 \mathrm{~W} / \mathrm{m} * K \ldots 2,88 \mathrm{~W} / \mathrm{m} * K$, based on the realized measurements. As seen in figure 4, the magnitude of the thermal conductivity has a significant effect on the distribution of temperature in the asphalt concrete. For higher values of the thermal conductivity, the temperature ratio increases the most rapidly, which is normally expected, resulting a more rapid approach of the balance temperature.

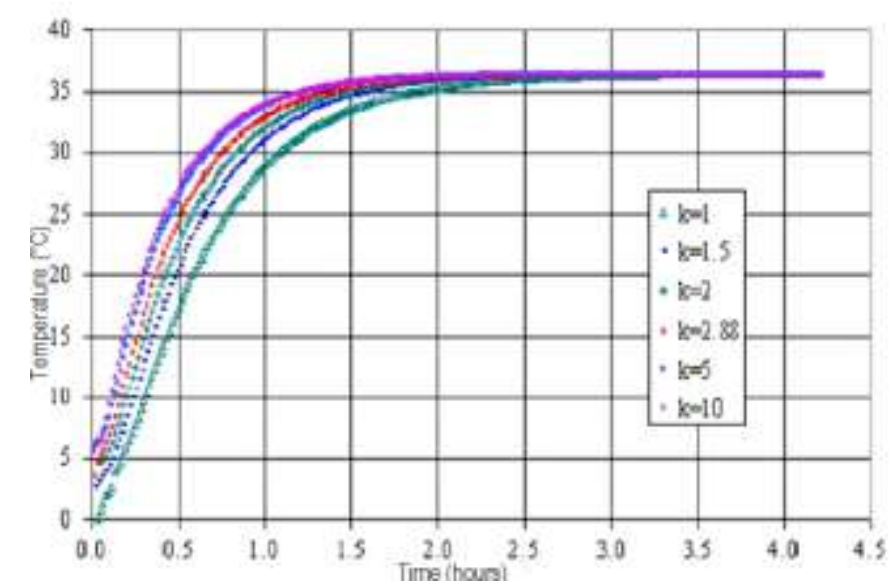

Figure 4. Predicted temperature for different thermal conductivities

\section{CONCLUSIONS}

The variation of temperature depending on the time was measured in different points on the sample of asphalt concrete in order to allow the implementation of a simulation model for the relation temperature - time with the help of the thermo-physical characteristics; this can be developed based on the logical chart presented in figure 5 with the help of a calculation program.

It was found that the analyses model could predict in a reliable way the variation of temperature and of distribution. In the same time, depending on the geometrical model of the samples, the sizes and thermo-physical characteristics have a significant influence on the relation time - temperature.

This paper presents the experiment for the case in which the temperature increases, the approach being applicable for a similar manner for very high or very low temperatures. 
The further validation of the technique proposed for determining the model temperature needs asphalt concrete dosages with different particle sizes, and different bitumen contents respectively, since all these thermo-physical characteristics have an important impact on the transfer heat.

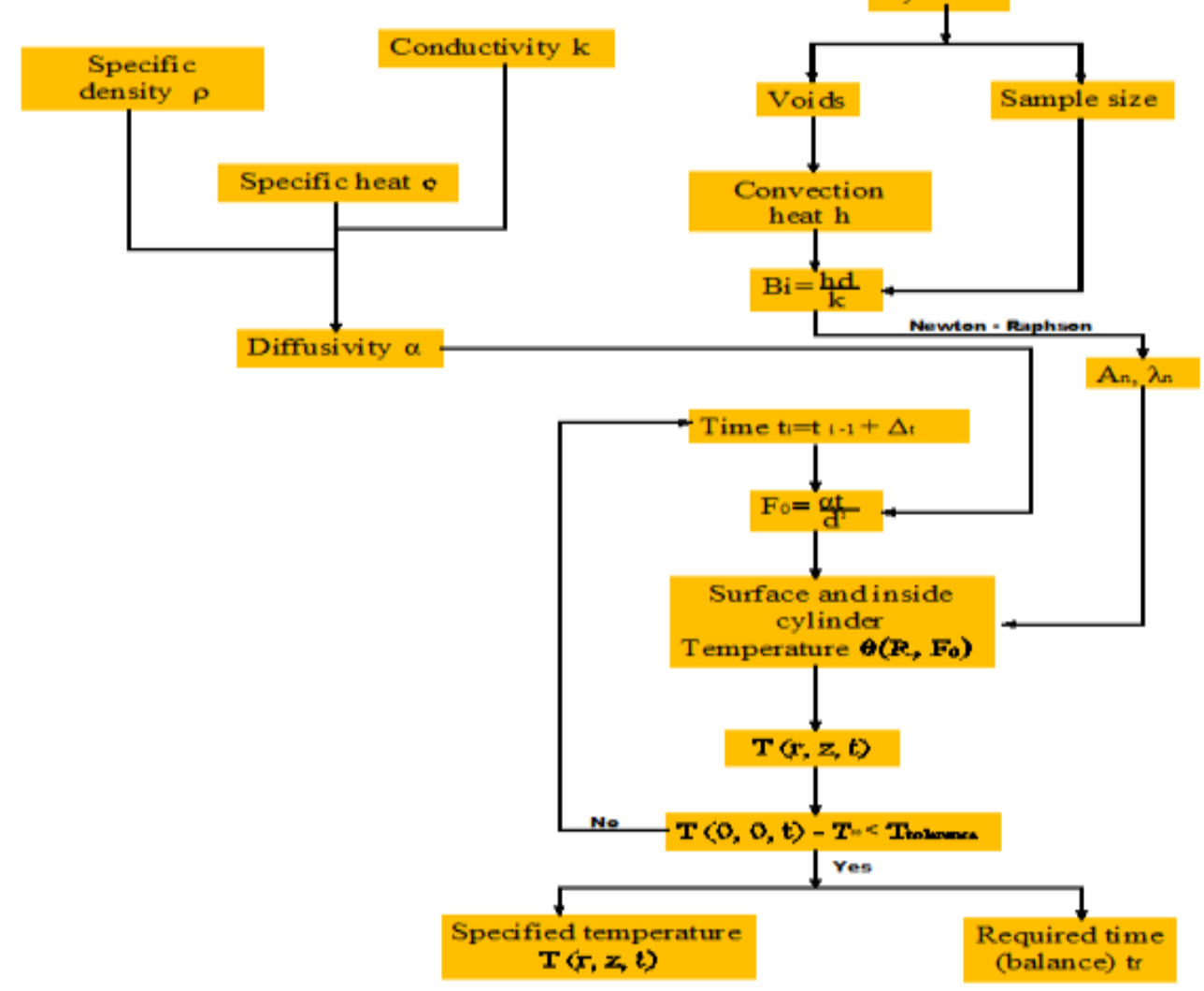

Figure 5. Organizational structure of temperature simulation (Q.Xu, M. Solaimanian)

\section{REFERENCES}

[1]. Arpaci SV, Kao SH, Selamet A. - Introduction to heat transfer. NJ; 1999.

[2]. Çengel YA. - Heat transfer-a practical approach. 2nd ed. McGraw-Hill Companies; 2002.

[3]. Emmanuel A., Didier D., Mohamed E., Bruno D. - Mesures fluxmétriques appliquées à l'étude des caractéristiques thermophysiques d'un mortier en cours de prise. XXII $^{\text {èmes }}$ rencontres universitaires de Génie Civil, Marne la Vallée, France 2004.

[4]. Luca J, Mrawia D. - New measurement of thermal properties of Superpave asphalt concrete. J Mater Civ Eng 2005;17:72-9.

[5]. Spalding DB. Pun WM.- A review of methods for predicting heat transfer coefficients for laminar uniform property boundary layer flow. Int J Heat Mass Transfer 
ROMANIAN JOURNAL

OF TRANSPORT INFRASTRUCTURE

Marc Paul, Costescu Ciprian

Research concerning the distribution of temperature in asphalt mixtures with the help of thermo-physical characteristics for laboratory tests

\section{9;5:239-49}

[6]. Zhao J. Ai X. Li YZ. - Transient temperature fields in functionally graded materials with different shapes under convective boundary conditions. Heat Mass Transfer 2007;43:1227-32.

[7]. Xu Q. Solaimanian M. - Measurement and evaluation of asphalt concrete thermal expansion and contraction. J Test Evalu, ASTM 2008:36. 